\title{
The pseudogap in hole-doped cuprates: possible insights from the Kondo effect
}

\author{
J. R. Cooper \\ Cavendish Laboratory, Department of Physics, University of Cambridge, \\ J.J. Thomson Avenue, CB3 OHE, United Kingdom
}

(Dated: December 23, 2021)

\begin{abstract}
The "states-non-conserving" fermion density of states (DOS), deduced from the specific heat of hole-doped cuprates, could arise from a Kondo or heavy fermion-like DOS being suppressed by antiferromagnetic spin fluctuations. The large Fermi surface predicted by band theory and observed experimentally, is still expected for zero pseudogap, but with an effective mass corresponding to a Kondo temperature $T_{K} \simeq 800 \mathrm{~K}$. A finite pseudogap could divide it into Fermi arcs. Theoretical results for the asymmetric Anderson model can account for the experimental Wilson ratio.
\end{abstract}

One of the unsolved questions for cuprate superconductors, in addition to the pairing mechanism and the linear temperature $(T)$ dependence of the electrical resistivity, is the origin of the pseudogap (PG) in samples with a certain number of holes $(p)$ per $\mathrm{CuO}_{2}$ unit. The PG lowers the superconducting condensation energy 1, 2], the superfluid density, e.g. 3], and an important practical property, the irreversibility field [4] where the electrical resistivity first becomes non-zero, even though the zero-field transition temperature initially stays high. Many researchers focus on a $T^{*}-p$ "phase diagram" and a $T^{*}(p)$ line, ascribed to the onset of a PG, that goes to zero as $p$ is increased to $p_{\text {crit }}[5]$. Careful experiments reveal anomalies in several physical properties at, or near $T^{*}[5]$, but the values of $T^{*}$ and the size of the anomalies are probably too small to account for the PG. For example the small [6] charge density wave (CDW) anomalies for under-doped (UD) samples may be linked [7] to the Fermi arcs, detected by angleresolved photoemission (ARPES), that are caused by the pre-existing PG. Abrupt changes in magnetic anisotropy of $\mathrm{YBa}_{2} \mathrm{Cu}_{3} \mathrm{O}_{6+x}$ (YBCO) crystals defining a nematic $T^{*}(p)$ line [8], that also extrapolates to zero at $p_{\text {crit }}$, are also small - typically $0.5 \%$ of the average in-plane spin susceptibility for $\Delta T \sim 50 \mathrm{~K}$. Here we retain the idea, reviewed along with many experimental facts in [9], that the PG is best described as a $T$-independent energy scale $E_{G}$ that varies strongly with $p$. We argue that it might be caused by low energy anti-ferromagnetic $(a f)$ spin fluctuations giving a deep dip in a Kondo or heavy fermion-like density of states (DOS) at the Fermi energy $\left(E_{F}\right)$.

Analysis of the specific heat of YBCO [10], $\mathrm{Bi}_{2} \mathrm{Sr}_{2} \mathrm{CaCu}_{2} \mathrm{O}_{8+x}$ (Bi2212) [2], at different oxygen levels $(x)$, and $\mathrm{La}_{2-y} \mathrm{Sr}_{y} \mathrm{CuO}_{4}$ (LSCO) [2]. in terms of a "states-non-conserving" V-shaped fermion DOS [1], centered at $E_{F}$, in an otherwise flat band, as sketched in Fig. 1(a), gives intriguing insights. It has has some deficiencies because (i) the large thermoelectric power (TEP) and its scaling with $E_{G}[11$, require electron-hole asymmetry, (ii) the detection of quantum oscillations and Fermi arcs implies that there is a small residual DOS at $E_{F}$ and (iii) $\mathrm{Y}^{89}$ NMR Knight shift data for over-doped (OD) Y(Ca)BCO 12] show a fall of up to $20 \%$ in the spin susceptibility, $\chi_{s}(T)$, at higher $T$ rather than the constant value expected for a flat band, there are also small deviations from flat band behavior for OD Bi2212 2]. On the other hand $\mathrm{Cu}$ NMR data for La-doped Bi2201 [13] seem to be consistent with a flat band. They also show that the PG is not affected by fields up to $44 \mathrm{~T}$, and that the residual DOS is much larger in Bi2201. The main features of this first-order phenomenological analysis are as follows.

A. $E_{G}$ falls linearly with $p$, as $J_{a f}\left(1-p / p_{\text {crit }}\right)$, disappearing abruptly at $p_{\text {crit }}=0.17-0.19[1]$, where $J_{a f} / k_{B}$ $=1200-1500 \mathrm{~K}$, is similar to the af interaction $J_{a f} / k_{B}=$ $1700 \mathrm{~K}[14]$ in the corresponding parent insulator with $p=0$ and $k_{B}$ is Boltzmann's constant.

B. Plots of $S_{e}(T, p)$ vs. $T$, where $S_{e}(T, p)$ is the nonphonon, electronic contribution to the entropy, are approximately linear and parallel at higher $T$, e.g. Fig. 1(b) of [2], and Fig. 5 of [10], or Figs. 1(SM) and 2(SM) of [15]. $S_{e}(T, p)$ curves for $p<p_{\text {crit }}$ have lower entropy at all $T$ and there is no sign of the entropy "coming back" up to at least $200 \mathrm{~K}$ for Bi-2212 and $300 \mathrm{~K}$ for YBCO. This behavior is consistent with a recent comprehensive NMR paper [16]. For fermions with an energy $(E)$ dependent DOS we expect $S_{e}(T) \alpha T \chi_{s}(T)$, because both depend on the number of states within the thermal window $\left|E-E_{F}\right| \lesssim 2 k_{B} T$. T $\chi_{s}(T)$ curves for Bi2212 are parallel up to $400 \mathrm{~K}$, see Fig. 13(b) of [2] or Fig. 3(SM) [15], as are plots of $T \chi_{s}(T)$ for $\mathrm{Y}_{0.8} \mathrm{Ca}_{0.2} \mathrm{Ba}_{2} \mathrm{Cu}_{3} \mathrm{O}_{6+x}$, also up to $400 \mathrm{~K}$ [17], after allowing for an $x$-independent Curie or Curie-Weiss term. The difference between a statesconserving CDW- or superconductor-like DOS and a Vshaped gap is shown by the results of model calculations of $S_{e}(T)$ in Fig. 4(SM) [15].

C. Plots of $S_{e}\left(T_{0}\right)$ vs. $p$ and $k_{B} T_{0} \chi_{s}\left(T_{0}\right) v s . p$ at temperatures $T_{0}$ show anomalously large increases of $\approx k_{B}$ and $0.3 \mu_{B}^{2}$ per added hole respectively [2], where $\mu_{B}$ is the Bohr magneton. For $p<p_{\text {crit }}$ this is caused by the $p$-dependence of the PG and is consistent with the Wilson ratio discussed later. For $p>p_{\text {crit }}$ they peak near $p=0.25$ before falling again, but this interesting result has only been established experimentally for LSCO [2].

D. $p_{\text {crit }}$ seems to be determined by the product of $J_{a f}$ and the DOS at high energy [2]. In contrast to points A to 
$\mathrm{C}$ this is not expected in the present picture because here $J_{a f}$ in the parent compound and the DOS for $E \gg E_{G}$ are not related.

It is widely accepted that the Hubbard model describes the basic physics of the cuprates, e.g. 18] and references therein, but it has not been solved rigorously at low $T$ and as far as we know does not account for points A to D above. Similar underlying physics occurs in the Friedel-Anderson model [19] and the Kondo effect [20, 21], where metals such as $\mathrm{Cu}$ or $\mathrm{Au}$ are alloyed with a small concentration $\left(c_{i m p}\right)$ of magnetic elements (impurities) such as Fe or Mn. For both Kondo alloys and heavy fermion compounds, the electronic specific heat coefficient, $\gamma \equiv d S_{e} / d T$, is strongly enhanced at low $T$, in Wilson's solution of the Kondo problem 22] it is given by

$$
\gamma_{i m p}=c_{i m p} \pi^{2} k_{B} w /\left(6 T_{K}\right)
$$

where $T_{K}$ is the Kondo temperature and $w=0.4128$ is the Wilson number. For impurities with spin $1 / 2$, and $T \ll T_{K}, \chi_{s}^{i m p}=c_{i m p}\left(g \mu_{B}\right)^{2} w /\left(4 k_{B} T_{K}\right)$, where $g \simeq 2$ is the $g$-factor and $\chi_{s}^{i m p} / \gamma_{i m p}=R_{W}=2 R_{0}$, in agreement with experiments on some Kondo alloys [21]. Here $R_{0}=3 \mu_{B}^{2} /\left(\pi^{2} k_{B}^{2}\right)$ is the Wilson ratio for non- or weaklyinteracting fermions.

For Bi2212, $\gamma(200)$ varies from 0.86 to $0.98 \mathrm{~mJ} / \mathrm{g}-$ at $/ \mathrm{K}^{2}$ for $0.095<p<0.22$ [2], and taking $c_{i m p}$ to be the concentration of $\mathrm{Cu}$ atoms, Eqn. [1 gives a first estimate for $T_{K} \simeq 800 \mathrm{~K}$. We note that such a single ion Kondo model works well for the heavy fermion compound $\mathrm{CePb}_{3}$ doped with La 23]. For the same range of $p$, $\chi_{s}(200)=2.18$ to $2.3810^{-4} \mathrm{emu} /$ mole Bi2212, see footnote 24] giving a Wilson ratio $\chi_{s} / \gamma=1.29$ to $1.34 R_{0}$. For $\mathrm{YBa}_{2} \mathrm{Cu}_{3} \mathrm{O}_{7}$, where there is some uncertainty from the $\mathrm{Cu}-\mathrm{O}$ chain contribution, and in the precise value of $\gamma(300), \chi_{s} / \gamma \simeq 1.2 R_{0}[2,25]$. So the experimental data is closer to $R_{0}$ rather than the Wilson value of $2 R_{0}$. This is not a limitation because Fig. 10.9 of Ref. [21] shows that for heavy fermion compounds such as $\mathrm{CeCu}_{2} \mathrm{Si}_{2}, \mathrm{CeCu}_{6}$ and $\mathrm{UPt}_{3}, \chi_{s} / \gamma \simeq R_{0}$, and as explained later, a ratio of 1.3 is consistent with the present picture. Numerical treatments of the $t-J$ model [26], derived from the 2D Hubbard model, give $\chi_{s} / \gamma \simeq R_{0}$ for $0.1<p<0.2$ and $T<J / k_{B}$. Large values of $T_{K}$ do occur for dilute alloys, e.g. AlMn where $T_{K}=600 \mathrm{~K}$ at low $T$ falling to $470 \mathrm{~K}$ near $300 \mathrm{~K}$ because of thermal expansion and the strong volume dependence of $T_{K}$ [27]. This reduces the $T$-dependence of $\chi_{s}(T)$ from the $1 /\left(T+T_{K}\right)$ law usually found for alloys with lower $T_{K}$ and less thermal expansion.

Here we consider three localized states, with occupancy 0,1 and 2 described by the asymmetric single orbital Anderson model. Figs. 1(b) and (c), taken from Fig. 5 of [28], show the spectral density for an isolated ion obtained by numerical renormalization group (NRG) calculations for $E_{0}=-U / 2$, the symmetric case, and various asymmetric cases, $-E_{0} / \Delta=4,3$ and $2 .\left|E_{0}\right|$ and $U-\left|E_{0}\right|>0$ are the energies required to transfer an electron from the singly-occupied state to the Fermi level of the conduction electrons at $E_{F}$, or from $E_{F}$ to the doubly-occupied state respectively. $U$, set equal to $4 \pi \Delta$ in [28], is the on-site $d-d$ Coulomb repulsion, $\Delta=\pi V^{2} N(E)$ [19], where $V$ is the hybridization energy between the $d$ state and the conduction electrons and $N(E)$ is their DOS for a given spin direction at the energy of the virtual bound state. The familiar triple peak structure in Fig. 1(b) has two side peaks arising from the localized level and a peak near $E_{F}$ from the Kondo resonance. The weak $p$ dependence of $\gamma$ and hence $T_{K}$ for OD Bi2212 and other OD cuprates implies that $E_{0}$ and $\Delta$ are also only weakly dependent on $p$.

As originally shown by Kondo 29] the central peak is caused by the af exchange interaction $-2 J_{i m p} \underline{S}_{i m p} \cdot \underline{S}_{e}$, with $J_{i m p}<0$, between the spin on the magnetic ion, $\underline{S}_{i m p}$ and the spin of a conduction electron $\underline{s}_{e}$. The $S_{i m p}^{+}$ and $S_{i m p}^{-}$operators do not commute, and because of this, higher order scattering processes contain the Fermi function for the conduction electrons. This leads to the logarithmic Kondo divergence in the scattering rate, responsible for the resistance minimum observed in dilute magnetic alloys and for the formation of the Kondo resonance at $E_{F}$. It is a many-body effect because it depends on the occupancy of many other conduction electron states.

The Kondo resonance has the same spatial symmetry as the electronic state of the localized level [20, [30]. This is why band-structures of heavy fermion materials, calculated using relatively standard methods, agree with the extremal orbits observed in quantum oscillation experiments, e.g. [31], because the band structures depend on this symmetry. But the effective masses $\left(m^{*}\right)$ are often very large, because they are determined by the energy width of the Kondo resonance. For the cuprates this implies that the Fermi surface is large, with the shape predicted by standard band theory, but $m^{*}$ is larger.

The effects of an applied magnetic field $(H)$ and, more generally, an exchange field, are important here. In dilute Kondo alloys, $H$ suppresses the Kondo effect because spin-flip scattering processes become inelastic in a magnetic field [20]. Early tunnelling studies where a fraction of a monolayer of $\mathrm{Fe}$ atoms was evaporated on the oxide layer of an $\mathrm{Al}-\mathrm{Al}_{2} \mathrm{O}_{3}-\mathrm{Al}$ junction [32] showed evidence for this "hole in the DOS" and it is indeed "non-statesconserving", see Fig. 9 of 32] shown in Fig. 5(SM) [15]. Experimentally [38, 39] and theoretically [40, 41] this requires $\mu_{B} H \gtrsim k_{B} T_{K}$. A problem here is that such a large value of $H, \approx k_{B} T_{K} / \mu_{B}$, is incompatible with the appropriate condition $E_{G} \simeq \mu_{B} H$ when $p \lesssim p_{\text {crit }}$ and $E_{G}$ is small.

This difficulty is absent for a molecular field in NRG calculations [42] for the two-band Hubbard model plus a ferromagnetic, predominantly Ising, exchange interaction $-2 J_{e x} S_{i}^{z} \cdot s^{z}$ between the localized magnetic mo- 


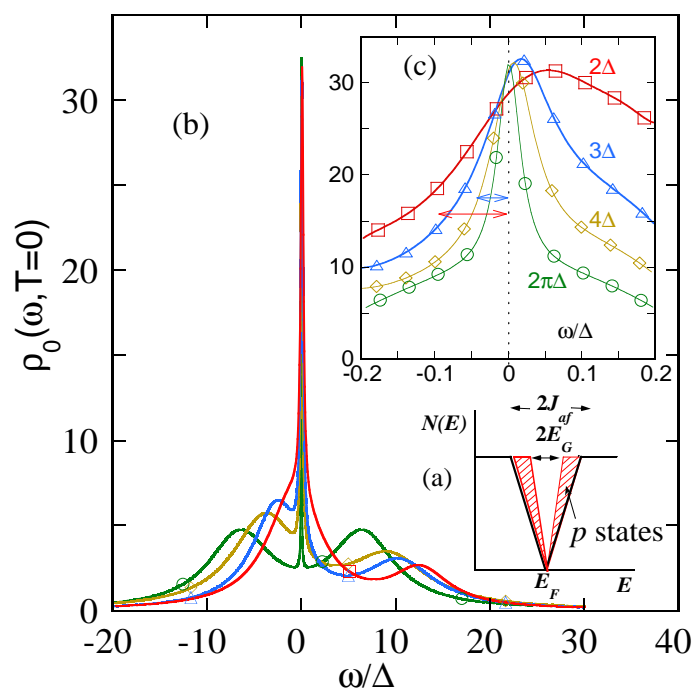

FIG. 1: Color online. (a) Fermion DOS [1] used to rationalize specific heat data. (b) and (c), taken from [28] with permission, triple peak structure in the spectral density $\rho_{0}(\omega, T=0)$ calculated using NRG theory for the single orbital, asymmetric Anderson model with $S_{i m p}=1 / 2$, for various values of $-E_{0} / \Delta=2 \pi, 4,3$ and 2 . Here we estimate $-E_{0} / \Delta=2.3$ for all the $\mathrm{Cu}$ ions in Bi2212. The horizontal arrows show $T_{K} / \Delta / 2$ for $-E_{0} / \Delta=2$ and 3 from 28 ]. Here the factor 2 ensures consistency between Eqn. 10 22] and [28], details are given in [15].

ments and the conduction electrons. Note that these calculations refer to a concentrated heavy fermion-like spin system. For $J_{e x}=0$ there are two side peaks from the narrow band, i.e. the localized magnetic states, and a central peak from the broad band, equivalent to the conduction electron band here. But as shown in 42 for large enough $J_{e x}$ there is a quadruple peak in the DOS curves, i.e. an extra dip at $E_{F}$, whose width is $\simeq 2 J_{e x}$. It is possible that $J_{e x}$ suppresses higher order spin-flip scattering processes, thereby suppressing the DOS at $E_{F}$. There appears to be no limitation regarding the ratio of $J_{e x} / k_{B} T_{K}$, in contrast to the effect of $H$ on Kondo alloys.

It is difficult to calculate $\Delta$ from first principles, even for noble-metal hosts, see 21], Chapt. 9.7. We attempted this by calculating the band-structure of the hypothetical compound $\mathrm{YBa}_{2} \mathrm{Zn}_{3} \mathrm{O}_{7}$, in which all lattice parameters are kept the same as for $\mathrm{YBa}_{2} \mathrm{Cu}_{3} \mathrm{O}_{7}$, using the Wien2K code [33]. In a sense this is equivalent to experimentalists using Lu compounds with a full $f$-shell as a reference for $\mathrm{Yb}$ compounds. The Brillouin zone is sketched in Fig. 6(SM)(a) [15] and dispersion curves shown in Fig. 6(SM)(b) [15]. The Zn $d$-levels are well below $E_{F}$ as shown by the the DOS vs. energy plots in Fig. 2 and there are approximately $10 d$-states per $\mathrm{Zn}$ atom, i.e. a full $d$ shell as expected from chemical arguments. In-plane states near $E_{F}$ do have a small amount

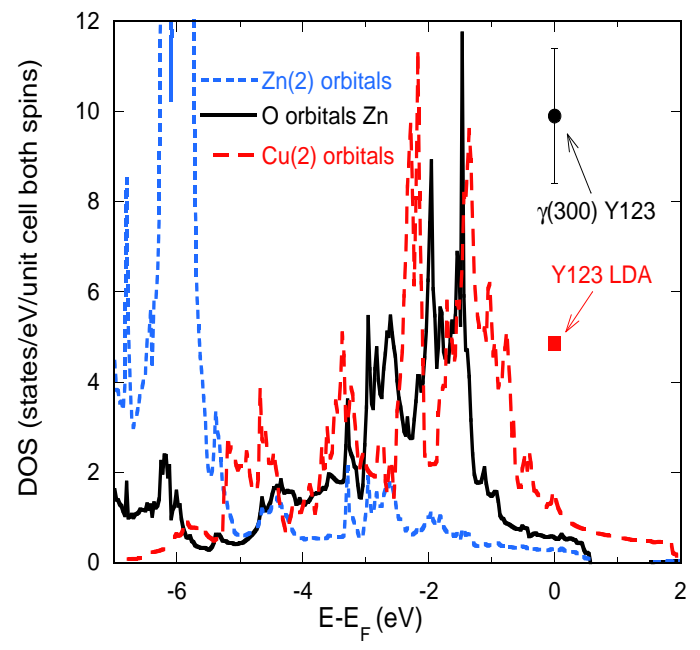

FIG. 2: Color online. Partial DOS vs. energy $E-E_{F}$; in-plane oxygen orbitals of $\mathrm{YBa}_{2} \mathrm{Zn}_{3} \mathrm{O}_{7}$, solid black line, $d$ orbitals for $\mathrm{Zn}(2)$ atoms in $\mathrm{YBa}_{2} \mathrm{Zn}_{3} \mathrm{O}_{7}$ and $\mathrm{Cu}(2)$ atoms in $\mathrm{YBa}_{2} \mathrm{Cu}_{3} \mathrm{O}_{7}$, short and long dashed lines respectively. Points show $N\left(E_{F}\right)$ of $\mathrm{YBa}_{2} \mathrm{Cu}_{3} \mathrm{O}_{7}$ from LDA and from $\gamma(300)$. When expressed as states $/ \mathrm{eV} / \mathrm{Cu}$ atom (both spins), $\gamma(300)$ for $\mathrm{YBa}_{2} \mathrm{Cu}_{3} \mathrm{O}_{7}$, gives $3.3 \pm 0.5$, the same within errors as $2.9 \pm 0.2$ states $/ \mathrm{eV} / \mathrm{Cu}$ atom (both spins) from $\gamma(200)$ of Bi2212.

of $d$ character but as shown in Fig. 2 this is only a few $\%$ of the DOS derived from the measured value of $\gamma$. In contrast, band structure calculations for $\mathrm{YBa}_{2} \mathrm{Cu}_{3} \mathrm{O}_{7}$ using the local density approximation (LDA) give substantial contributions to $N\left(E_{F}\right)$ from the $\mathrm{Cu} 3 d$ orbitals forming the $\mathrm{Cu}-\mathrm{O}_{2}$ plane bands. The full LDA DOS for $\mathrm{YBa}_{2} \mathrm{Cu}_{3} \mathrm{O}_{7}$ (not shown) agrees well with earlier work [34] and $\gamma(300)$ is only moderately enhanced over the LDA value, see Fig. 2. But $N\left(E_{F}\right)$ from the in-plane oxygen bands and the in-plane $\mathrm{Zn}$ atoms in $\mathrm{YBa}_{2} \mathrm{Zn}_{3} \mathrm{O}_{7}$ is much smaller, so in the present picture the measured values of $\gamma$ for YBCO and Bi2212 arise from the Kondo effect - Eqn. 1. Fig. 6(SM)(c) 15] shows the Fermi surface of $\mathrm{YBa}_{2} \mathrm{Zn}_{3} \mathrm{O}_{7}$, with two quasi-cylindrical sheets of similar size from the bands crossing $E_{F}$ between $\mathrm{S}$ and $\mathrm{X}$ and $\mathrm{S}$ and $\mathrm{Y}$, and an open surface, from the band crossing $E_{F}$ between $Y$ and G. From Fig. 6(SM)(b) [15] the average value of $E_{F}$ for the two quasi-cylindrical bands of the $\mathrm{Zn}$ compound is $0.54 \mathrm{eV}$ relative to the top of the band. The average value of $k_{F}$ in the $\mathrm{S}-\mathrm{X}-\mathrm{Y}$ plane is $0.35 \pi / a$ where $a=0.382 \mathrm{~nm}$ is the in-plane lattice spacing, giving $p$ for the two cylindrical bands which agrees well with the more precise value obtained by integrating the DOS plots in Fig. 2, for the in-plane $\mathrm{O}(2), \mathrm{O}(3)$ and $\mathrm{Zn}(2)$ atoms. Namely $p=2 \times 0.19 /$ unit cell, in surprisingly good agreement with empirical experimental estimates for $\mathrm{YBa}_{2} \mathrm{Cu}_{3} \mathrm{O}_{7} 35$ 37. It would be interesting to see whether this procedure works for the two-chain compound $\mathrm{YBa}_{2} \mathrm{Cu}_{4} \mathrm{O}_{8}$. 
The complex band structure below $\approx-1 \mathrm{eV}$ shown in Figs. 2 and 6(SM)(b) [15] prevents us estimating $\Delta$ from first principles, so initially we set $\Delta=1 \mathrm{eV}$, a typical value for $3 d$ impurities in noble metals [19, 20]. But, as explained in more detail in [15], we can account for the experimental value of the Wilson ratio if $E_{0} / \Delta=-2.3$, because for this value, linear interpolation of the data in Table 1 of 28] gives the occupancy of the localized level, $n_{0}=0.81$ and an effective moment of $0.81 \mu_{B}$. This reduces the susceptibility by $0.81^{2}$ and the Wilson ratio from the value 2 expected for an ion with $S=1 / 2$ to 1.31 , in good agreement with experiment. Theoretical $S_{e}(T)$ curves were obtained by integrating the specific heat data in Fig. 4 of [28] divided by $T$. For $T_{K}=800 \mathrm{~K}, \gamma$ increases by a factor 1.19 between $T=200 \mathrm{~K}$ and $T=0$, so the initial estimate of $T_{K}=800 \mathrm{~K}$ from $\gamma(200)$ of Bi2212 is reduced to $672 \mathrm{~K}$. Theoretical curves for representative samples of $\mathrm{Bi} 2212$, one with no $\mathrm{PG}, p=0.19$ and one with a $\mathrm{PG}$, are linear over the measured range of $T \leq 220 \mathrm{~K}$ as shown in Fig. 7(SM) [15]. In contrast those for $\mathrm{YBa}_{2} \mathrm{Cu}_{3} \mathrm{O}_{6.97}$ with no $\mathrm{PG}$ and $\mathrm{YBa}_{2} \mathrm{Cu}_{3} \mathrm{O}_{6.48}$ with $E_{G} / k_{B}=320 \mathrm{~K}$, in Fig. 8(SM) 15], both having $T_{K}=775 \mathrm{~K}$, show deviations from linearity above $200 \mathrm{~K}$. But these do not rule out the present picture because they could arise from a modest decrease in $T_{K}$ caused by thermal expansion, or because the $\mathrm{Cu}$ atoms in the $\mathrm{CuO}$ chains and the $\mathrm{CuO}_{2}$ planes are inequivalent.

Hence for $\mathrm{Bi} 2212 T_{K}=672 \mathrm{~K}$ and for $E_{0} / \Delta=-2.3$, $k_{B} T_{K} / \Delta=0.073$, and $\Delta=0.79 \mathrm{eV}$. The observation of significant $g$-factor anisotropy in YBCO [6] and references therein, supports the present localized picture. Here the concept of valency is meaningful, the $\mathrm{Cu}^{2+}\left(d^{9}\right)$ state will be dominant and for $-E_{0}=2.3 \Delta$ there is a certain admixture of $\mathrm{Cu}^{3+}\left(d^{8}\right)$. The $d^{10}$ and $d^{9}$ states are analogous to those in heavy fermion $\mathrm{Yb}$ compounds with 0 or 1 hole in the $4 f$ shell, which implies that all the $\mathrm{Cu} d$ electrons are contributing to $U$. As shown in Fig. 1(c) the half-width-half-maximum (HWHM) values of the spectral density at $\omega<0$ for $-E_{0} / \Delta=3$ and 2 are $\simeq 0.076 \Delta$ and $0.135 \Delta$ respectively giving $0.117 \Delta$ for $-E_{0} / \Delta=2.3$. The carrier density in the resonance $n_{K}$, obtained by assuming that it has a Lorentzian shape for $\omega<0$ and normalizing to the area of the lowest broad peak in Fig. 1 (b) that contains $\simeq 1$ electron, agrees well with $\left(1-n_{0}\right) / 2=0.095$. For $\Delta=0.79 \mathrm{eV}$ the HWHM is $T_{L}=1072 \mathrm{~K}$. When transformed in the usual way into $\underline{k}-$ and then by Fourier transform into $\underline{r}-$ space, and in the absence of a $P G$, the carrier density decays as $n_{K} \exp (-r / R)$ with a range $R=\frac{\hbar v_{F}}{k_{B} T_{L}}$. The resonance is centered at the Fermi level and because of the sharpness of the Fermi surface, the Fourier transform could also give rise to Friedel or Rudermann-Kittel-Kasuya-Yosida type oscillations in $n_{k}(r)$, that could also be important but are not considered here. The Fermi velocity $v_{F}=$ $1.610^{7} \mathrm{~cm} / \mathrm{sec}$, estimated for a cylindrical surface containing $1+p$ holes with $m^{*}=5.2 m_{e}$ [43], agrees well with nodal ARPES data for UD and optimally-doped (OP) crystals of the single layer cuprate $\mathrm{Hg} 1201$ shown in Fig. 2(b) of [44], 1.7, 2.2 and $2.010^{7} \mathrm{~cm} / \mathrm{sec}$ for UD70, UD80 and OP98. This agreement supports our statement that $T_{K}$ and hence $m^{*}$ vary little with $p$.

The above values of $v_{F}$ and $T_{L}$ give $R=2.83 a$. From the previous spatial symmetry argument, $n_{K}$ will have $\left|d_{x^{2}-y^{2}}\right|$ symmetry and be larger in the $(0,1)$ and $(1,0)$ directions, where af spin fluctuations cause neighboring spins to be anti-parallel over a certain spatial range, $\xi$. A typical value for $p=0.1$ is $\xi=0.9 \mathrm{~nm}$, i.e. $2-3 a$ [14]. If $\xi=2 a$ then only the 4 nearest neighbors have correlated spins. Their af Kondo screening "clouds" will give a ferromagnetic conduction electron spin polarization at the central site. Including the effect of the PG on both $n_{k}$ and $R$ (by self-consistently representing the PG by a narrower negative Lorentzian) reduces $n_{k}$ at the central site by a factor $\simeq 2$, giving a magnetic moment from the 4 overlapping Kondo "clouds" of $2 \times 0.095 \mathrm{x}$ $\exp (-1 / 2.83) \mu_{B}=0.133 \mu_{B}$. It is difficult to prove that this spin polarization is equivalent to $J_{e x}$ in [42], but, for $\chi_{s}=2.310^{-4} \mathrm{emu} /$ mole-Bi2212, a spin polarization of $0.133 \mu_{B} / \mathrm{Cu}$ is produced by a field $H$ where $\mu_{B} H / k_{B}=$ $433 \mathrm{~K}$, so $J_{e x} / k_{B}=866 \mathrm{~K}$. From Fig. 2 of [42] the half energy gap, equivalent to $E_{G}$ here, is $\simeq J_{e x}$, in reasonable agreement with the experimental value $E_{G} / k_{B} \simeq 600 \mathrm{~K}$ for $p=0.1$. Furthermore, the imaginary part of the self energy is unusual and typical of a "bad metal" 42] in agreement with experiments on the cuprates. However, more work is needed to see whether $J_{e x}$ falls linearly with $p$ and whether calculations allowing for asymmetry in the DOS about $E_{F}$ on a scale of $J_{e x}$, i.e. $E_{G}$, would account for the strong $p$-dependence of the TEP and its scaling with $k_{B} T / E_{G}$ [11, 25], shown in Fig. 9(SM) [15].

Alternatively, in a related picture, the effect of the $a f$ fluctuations, i.e. the interactions between neighboring $\mathrm{Cu}$ spins, could be considered directly, without appealing to the work of Ref. [42]. These will reduce the entropy of the $\mathrm{Cu}$ spins and hence that available for the Kondo resonance. Key theoretical questions are (a) can they cause a deep PG even when the interaction energy $(W)$ is much smaller than $k_{B} T_{K}$ and (b) does $W$ fall linearly with $p$ and become very small for $p \geq p_{\text {crit }}$ ? Experimental evidence for (b) is given by ratio of the ${ }^{63} \mathrm{Cu}$ and ${ }^{17} \mathrm{O}$ NMR relaxation rates $T_{1}^{-1}$ [9] which is a measure of the strength of low frequency spin fluctuations for which the effect of the PG on $\chi_{s}$ cancels out. It does indeed fall linearly with $p$, approaching zero at $p_{\text {crit }}$. Analysis [45] of neutron scattering data shows the same decrease for $a f$ spin fluctuations of energy below $50 \mathrm{meV}$, so on the time scale of the Kondo resonance, with $k_{B} T_{K} \approx 60 \mathrm{meV}$, they will provide a molecular field that is nearly static.

Within the usual localized picture $W$ arises from superexchange via completely occupied or completely empty oxygen $2 p$ states. For a band with Fermi energy $E_{F}$, we should consider Wannier functions that 
have a lifetime of order $\hbar / E_{F} . E_{F}$ will increase with $p$, thereby reducing $W$, which could account for point (b) above. As sketched for example in Fig. 13 of [25], only those parts of the large Fermi surface spanned by the $a f$ wave vector $\underline{Q} \approx\left(\frac{\pi}{a}, \frac{\pi}{a}\right)$, will be affected by af fluctuations. This accounts for the Fermi arcs, where $E_{G}=0$, seen by ARPES [46] and scanning tunnelling microscopy (STM) [47]. It also gives a strong energy dependence of the scattering rate responsible for the TEP [25], see also [48], and its scaling with $k_{B} T / E_{G}$ shown in [25] and Fig. 9(SM) [11, 15].

Evidence for an energy scale $\approx 60 \mathrm{meV}$ in the many spectroscopic studies of cuprates above $T_{c}$ would be a good experimental test of the present picture. ARPES data do show structure near $50 \mathrm{meV}$, e.g. [44] that is presently ascribed to other factors. However, we are dealing with a many-body effect, so comparison of ARPES, STM [47] and optical data [49, 50] for cuprates with those for heavy fermion compounds as well as with theory could be informative. Another test would the apparent correlation between the $T^{1}$ term in the electrical resistivity and the superfluid density [51] in OD compoumds. One prediction of the present approach is that $m^{*}$ and related properties of OD Tl2201 crystals could be strongly pressure dependent since for classical Kondo alloys the volume $(V)$ dependence of $T_{K}$ is large, $-\operatorname{dln} T_{K} / \operatorname{dln} V=$ 16-18 [27, 52].

In summary, guided by the unusual behavior of the electronic entropy revealed by specific heat measurements [1, 2, 10], we propose that the pseudogap is the energy scale over which a Kondo-like enhanced DOS at the Fermi energy is suppressed by af spin interactions.

The author would like to thank A. Carrington, who also calculated the band structure of $\mathrm{YBa}_{2} \mathrm{Zn}_{3} \mathrm{O}_{7}$, J. L. Tallon and V. Zlatić for helpful suggestions and discussions and to acknowledge a long and happy collaboration with the late Dr. J. W. Loram.

[1] J. W. Loram, K. A. Mirza, J. R. Cooper and J. L. Tallon, Specific heat evidence on the normal state pseudogap, J. Phys. Chem. Solids 59, 2091-94 (1998).

[2] J. W. Loram, J. Luo, J. R. Cooper, W. Y. Liang and J. L. Tallon, Evidence on the pseudogap and condensate from the electronic specific heat, J. Phys. Chem. Solids 62, 59-64 (2001).

[3] W. Anukool, S. Barakat, C. Panagopoulos and J. R. Cooper, Effect of hole doping on the London penetration depth in $\mathrm{Bi}_{2.15} \mathrm{Sr}_{1.85} \mathrm{CaCu}_{2} \mathrm{O}_{8+\delta}$ and $\mathrm{Bi}_{2.1} \mathrm{Sr}_{1.9} \mathrm{Ca}_{0.85} \mathrm{Y}_{0.15} \mathrm{Cu}_{2} \mathrm{O}_{8+\delta}$, Phys. Rev. B 80024516 (2009).

[4] J. R. Cooper, J. W. Loram, J. D. Johnson, J. W. Hodby, and Chen Changkang, 3D XY scaling of the irreversibility line of $\mathrm{YBa}_{2} \mathrm{Cu}_{3} \mathrm{O}_{7}$ crystals, Phys. Rev. Lett. 79, 1730 (1997).

[5] B. Keimer, S. A. Kivelson, M. R. Norman, S. Uchida and
J. Zaanen, From quantum matter to high-temperature superconductivity in copper oxides, Nature 519 179-186 (2015).

[6] I. Kokanović and J. R. Cooper, Magnetic susceptibility of $\mathrm{YBa}_{2} \mathrm{Cu}_{3} \mathrm{O}_{6+x}$ crystals: Unusual Curie behavior and small contributions from charge density waves, Phys. Rev. B 94 075155-11 (2016).

[7] R. Comin, A. Frano, M. M. Yee, Y. Yoshida, H. Eisaki, E. Schierle, E.Weschke, R. Sutarto, F. He, A. Soumyanarayanan, Y. He, M. Le Tacon, I. S. Elfimov, J. E. Hoffman, G. A. Sawatzky, B. Keimer, and A. Damascelli, Charge order driven by Fermi-arc instability in $\mathrm{Bi}_{2} \mathrm{Sr}_{2-x} \mathrm{La}_{x} \mathrm{CuO}_{6+\delta}$, Science 343, 390-392 (2014).

[8] Y. Sato, S. Kasahara1, H. Murayama, Y. Kasahara, E.G. Moon, T. Nishizaki, T. Loew, J. Porras, B. Keimer, T. Shibauchi and Y. Matsuda, Thermodynamic evidence for a nematic phase transition at the onset of the pseudogap in $\mathrm{YBa}_{2} \mathrm{Cu}_{3} \mathrm{O}_{y}$, Nature Physics, 131074 (2017).

[9] J. L. Tallon and J. W. Loram, The doping dependence of $\mathrm{T}^{*}$ - what is the real high-T-c phase diagram?, Physica CSuperconductivity and its Applications 349 53-68 (2001).

[10] J. W. Loram, K. A. Mirza, J. R. Cooper and W. Y. Liang, Electronic specific heat of $\mathrm{YBa}_{2} \mathrm{Cu}_{3} \mathrm{O}_{6+x}$ from 1.8 to 300 K, Phys. Rev. Lett. 71 1740-1743 (1993).

[11] J. R. Cooper, H. Minami, V. W. Wittorff, D. Babić and J. W. Loram, Effect of the normal state gap on the thermoelectric power, irreversibility line and c-axis resistivity of $\mathrm{YBa}_{2} \mathrm{Cu}_{3} \mathrm{O}_{7-\delta}$, Physica C 341 855-858 (2000).

[12] G. V. M. Williams, J. L. Tallon, R. Michalak and R. Dupree, NMR studies of overdoped $\mathrm{Y}_{1-x} \mathrm{Ca}_{x} \mathrm{Ba}_{2} \mathrm{Cu}_{3} \mathrm{O}_{7-\delta}$, Phys. Rev. B 57 8696-8701 (1998).

[13] S. Kawasaki, C. T. Lin, P. L.Kuhns, A. P. Reyes and G. Zheng, Carrier-concentration dependence of the pseudogap ground state of superconducting $\mathrm{Bi}_{2} \mathrm{Sr}_{2-x} \mathrm{La}_{x} \mathrm{CuO}_{6+\delta}$ revealed by $\mathrm{Cu}-63, \mathrm{Cu}-65-\mathrm{Nuclear}$ Magnetic Resonance in very high magnetic fields, Phys. Rev. Lett. 105137002 (2010).

[14] J. Rossat-Mignod, L. P. Regnault, C. Vettier, P. Bourges, P. Burlet, J. Bossy, J. Y. Henry and G. Lapertot, Spin dynamics in the high $\mathrm{T}_{c}$ system $\mathrm{YBa}_{2} \mathrm{Cu}_{3} \mathrm{O}_{6+x}$, Physica B 180-181 383-388 (1992).

[15] See Supplemental Material(SM) for additional Figures and explanations.

[16] J. Nachtigal, M. Avramovska, A. Erb, D. Pavičević, R. Guehne and J. Haase, Temperature-independent cuprate pseudogap from planar oxygen NMR, Condens. Matter 5, 66-84 (2020).

[17] S. H. Naqib, J. R. Cooper, and J. W. Loram, Effects of $\mathrm{Ca}$ substitution and the pseudogap on the magnetic properties of $\mathrm{Y}_{1-x} \mathrm{Ca}_{x} \mathrm{Ba}_{2} \mathrm{Cu}_{3} \mathrm{O}_{7-\delta}$, Phys. Rev. B 79, 104519-8 (2009).

[18] W. Wu, M. S. Scheurer, S. Chatterjee, S. Sachdev, A. Georges and M. Ferrero, Pseudogap and Fermi-surface topology in the two dimensional Hubbard model, Phys. Rev. X 8021048 (2018).

[19] P. W. Anderson, Localized magnetic states in metals, Phys. Rev. 124, 41 (1961).

[20] G. Grüner Experimental evidence for many-body effects in dilute alloys, Adv. Phys. 23 941-1023 (1974).

[21] A. C. Hewson, "The Kondo problem to heavy fermions", Cambridge University Press, Cambridge, U.K. (1993).

[22] K. G. Wilson, Renormalization group- critical phenomena and Kondo problem, Rev. Mod. Phys. 47 773-840 
(1975)

[23] C. L. Lin, A. Wallash, J. E. Crow, T. Mihalisin and P. Schlottmann, Heavy-fermion behavior and the single-ion Kondo model, Phys. Rev. Lett. 58 1232-1235 (1987).

[24] The measured susceptibility of Bi2212 at $300 \mathrm{~K}$, is 1.1 to $1.310^{-4} \mathrm{emu} / \mathrm{mole}-\mathrm{Bi} 2212$ [2] for $0.095<p<0.22$, giving $\chi_{s}=2.18$ to $2.3810^{-4} \mathrm{emu} / \mathrm{mol}-\mathrm{Bi} 2212$ after corrections for core diamagnetism and Van Vleck orbital paramagnetism. These are respectively $-1.9510^{-4} \mathrm{emu} / \mathrm{mol}-$ Bi2212 [53] and $0.86610^{-4} \mathrm{emu} / \mathrm{mol}-\mathrm{Bi} 2212$, using the $\mathrm{Cu}-\mathrm{O}_{2}$ plane value for YBCO [54].

[25] J. R. Cooper and J. W. Loram, Some correlations between the thermodynamic and transport properties of high $T_{c}$ oxides in the normal state, J. Phys. I France 6 2237-2263 (1996).

[26] J. Jaklić and P. Prelovšek, Finite-temperature properties of doped antiferromagnets, Adv. Phys. 49 1-92 (2000).

[27] J. R. Cooper and M. Miljak, Single impurity behaviour and interaction effects in the magnetic susceptibility of AlMn and AlCr alloys, J. Phys. F: Met. Phys. 621 512164 (1976).

[28] T. A. Costi, A. C. Hewson and V. Zlatić, Transport coefficients of the Anderson model via the numerical renormalization group, J. Phys.: Condens. Matter 6 2519-2558 (1994).

[29] J. Kondo, Resistance minimum in dilute magnetic alloys, Prog. Theor. Phys. 32 37-49 (1964).

[30] A. Blandin, Magnetic impurities in metals, J. Appl. Phys. 39 1285-1294 (1968).

[31] A. Pourret, M-T. Suzuki, A. P. Morales, G. Seyfarth, G. Knebel, D. Aoki, and J. Flouquet, Fermi surfaces in the antiferromagnetic, paramagnetic and polarized paramagnetic states of $\mathrm{CeRh}_{2} \mathrm{Si}_{2}$ compared with quantum oscillation experiments, J. Phys. Soc. Jpn. 86, 084702-7 (2017).

[32] S. Bermon, D. E. Paraskevopoulos and P. M. Tedrow, Ultra-high magnetic field study of the Kondo-type zerobias conductance peak in magnetically doped metalinsulator-metal tunnel junctions, Phys. Rev. B 17 21102123 (1978).

[33] P. Blaha, K. Schwarz, G. K. H. Madsen, D. Kvasnicka and J. Luitz, "WIEN2K, An augmented plane wave + local orbitals program for calculating crystal properties", Karlheinz Schwarz, Techn. Universität Wien, Austria, ISBN 3-9501031-1-2 (2001).

[34] W. E. Pickett, Electronic structure of the hightemperature oxide superconductors, Rev. Mod. Phys. 61 434-511 (1989).

[35] M. R. Presland, J. L. Tallon, R. G. Buckley, R. S. Liu and N. E. Flower, General trends in oxygen stoichiometry on $T_{c}$ in $\mathrm{Bi}$ and $\mathrm{Tl}$ superconductors, Physica C 176 95-105 (1991).

[36] R. Liang, D. A. Bonn and W. N. Hardy, Evaluation of $\mathrm{CuO}_{2}$ plane hole doping in $\mathrm{YBa}_{2} \mathrm{Cu}_{3} \mathrm{O}_{6+x}$ single crystals, Phys. Rev. B 73 180505-180508(R) (2006).

[37] S. D. Obertelli, J. R. Cooper and J. L. Tallon, Systematics in the thermoelectric power of high T-c oxides, Phys. Rev. B 46 14928(R) (1992).

[38] P. Monod, Magnetic field dependence of the Kondo resistivity minimum in $\mathrm{CuFe}$ and CuMn alloys, Phys. Rev. Lett. 19 1113-1156 (1967).

[39] H. Rohrer, High-field magnetoresistance of dilute $\mathrm{Cu}-\mathrm{Mn}$ and $\mathrm{Cu}-\mathrm{Fe}$ alloys, Journ. Applied Phys. 40, 1472-1473 (1969).
[40] B. Horvatić and V. Zlatić, Magnetic field effects for the asymmetric Anderson Hamiltonian, Phys. Rev. B 30 6717-6731 (1984).

[41] T. A. Costi, Kondo effect in a magnetic field and the magnetoresistivity of Kondo alloys, Phys. Rev. Lett. 85 1504-1507 (2000).

[42] T. A. Costi and A. Liebsch, Quantum phase transition in the two-band Hubbard model, Phys. Rev. Lett. 99 236404-4 (2007).

[43] A. F. Bangura, P. M. C. Rourke, T. M. Benseman, M. Matusiak, J. R. Cooper, N. E. Hussey, and A. Carrington, Fermi surface and electronic homogeneity of the overdoped cuprate superconductor $\mathrm{Tl}_{2} \mathrm{Ba}_{2} \mathrm{CuO}_{6+\delta}$ as revealed by quantum oscillations, Phys. Rev. B 82 140501(R) (2010).

[44] S. A. Sreedhar, A. Rossi, J. Nayak, Z. W. Anderson, Y. Tang, B. Gregory, M. Hashimoto, D.-H. Lu, E. Rotenberg, R. J. Birgeneau, M. Greven, M. Yi and I. M. Vishik, Three interaction energy scales in the single-layer highTc cuprate $\mathrm{HgBa}_{2} \mathrm{CuO}_{4+\delta}$, Phys. Rev. B 102, 205109 (2020).

[45] J. G. Storey, J. L. Tallon and G. V. M. Williams, Pseudogap ground state in high temperature superconductors, Phys. Rev. B 78 140506(R) (2008).

[46] S. Chen, M. Hashimoto, Y. He, D. Song, K-J. Xu, J.-F. He, T. P. Devereaux, H. Eisaki, D-H. Lu, J. Zaanen and Z-X. Shen, Incoherent strange metal sharply bounded by a critical doping in Bi2212, Science 366, 1099-1102 (2019).

[47] K. Fujita, C. K. Kim, I. Lee, J. Lee, M. H. Hamidian, I. A. Firmo, S. Mukhopadhyay, H. Eisaki, S. Uchida, M. J. Lawler, E-A. Kim and J. C. Davis, Simultaneous transitions in cuprate momentum-space topology and electronic symmetry breaking, Science 344 612-616 (2014).

[48] G. Hildebrand, T. J. Hagenaars, W. Hanke, S. Grabowski and J. Schmalian, Effects of electronic correlations on the thermoelectric power of the cuprates, Phys. Rev. B 56 R4317-4320 (1997).

[49] D. N. Basov and T. Timusk, Electrodynamics of high- T $_{c}$ superconductors, Rev. Mod. Phys. 77721 (2005).

[50] S. Tajima, Optical studies of high-temperature superconducting cuprates, Rep. Prog. Phys. 79094001 (2016).

[51] M. Čulo, C. Duffy, J. Ayres, M. Berben, Y.-T. Hsu, R. D. H. Hinlopen, B. Bernáth and N. E. Hussey, Possible superconductivity from incoherent carriers in overdoped cuprates, SciPost Phys. 11, 012 (2021).

[52] J. S. Schilling and W. B. Holzapfel, Effect of pressure on the Kondo temperature of $\mathrm{Cu}$ :Fe-existence of a universal resistivity curve, Phys. Rev. B 8 1216-1237 (1973).

[53] P. W. Selwood, "Magnetochemistry", Interscience Publishers (1964).

[54] F. Mila and T. M. Rice, Analysis of magnetic resonance experiments in $\mathrm{YBa}_{2} \mathrm{Cu}_{3} \mathrm{O}_{7}$, Physica C 157 561570 (1989).

[55] H.-U. Desgranges and K. D. Schotte, Specific heat of the Kondo model, Phys. Lett. 91A 240 (1982).

[56] V. T. Rajan, Magnetic susceptibility and specific heat of the Coqblin-Schrieffer model, Phys. Rev. Lett. 51 308311 (1983).

[57] J. W. Loram, K. A. Mirza and J. R. Cooper, "IRC Research Review", pp 75-97, Ed. W. Y.Liang, University of Cambridge, Cambridge, U.K. (1998). 